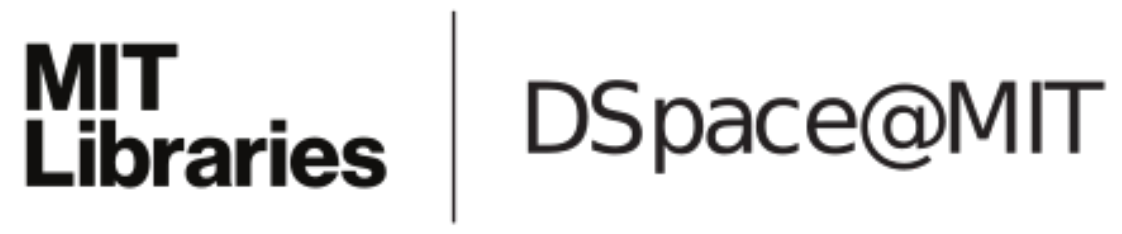

\author{
MIT Open Access Articles
}

Effect of red blood cells on platelet aggregation

The MIT Faculty has made this article openly available. Please share how this access benefits you. Your story matters.

Citation: Pivkin, I., P. Richardson, and G. Karniadakis. "Effect of red blood cells on platelet aggregation." Engineering in Medicine and Biology Magazine, IEEE 28.2 (2009): 32-37. ( 2009 IEEE.

As Published: http://dx.doi.org/10.1109/MEMB.2009.931788

Publisher: Institute of Electrical and Electronics Engineers

Persistent URL: http://hdl.handle.net/1721.1/60238

Version: Final published version: final published article, as it appeared in a journal, conference proceedings, or other formally published context

Terms of Use: Article is made available in accordance with the publisher's policy and may be subject to US copyright law. Please refer to the publisher's site for terms of use. 


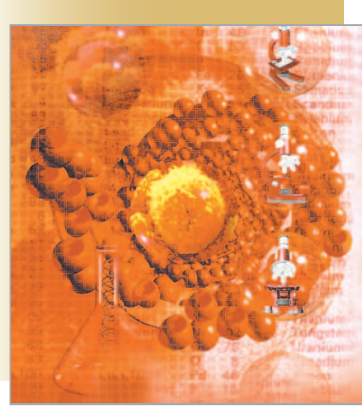

\title{
Effect of Red Blood Cells on Platelet Aggregation
}

\author{
Enhanced Diffusivity of Platelets
}

BY IGOR V. PIVKIN, PETER D. RICHARDSON, AND GEORGE EM KARNIADAKIS

\section{T}

he blood cell that plays a central role in the normal clotting process is the platelet. Blood platelets were first discovered by Donne in 1842 [1], but 40 years later, Bizzozero [2] recognized the importance of platelet clumping in the blood clotting process. It is now well established that platelet aggregation is not only important for primary hemostasis but when exaggerated can also lead to the formation of occlusive thrombi [3], which form at sites of atherosclerotic plaque rupture, resulting in a heart attack, stroke, or sudden death.

Platelet aggregation involves multiscale processes; at nanoscale, many phenomena are known to cell biologists, but the inclusion into models of flow-modulated phenomena such as cell adhesion and aggregation at the micron-scale needs to be developed. At still larger scale, using some simplifications of the nanoscale effects, this may be extended to a detailed analysis of the (platelet) particulate flow, cell (leukocyte, monocyte, and tumor) migration at endothelial cell layers from flowing blood, the embolization of mural thrombi, and for the in vitro experiments relations for the variability of measured bleeding times.

Indeed, there is a prospect of coupling multiscale representations of blood flow, ranging from a quasi one-dimensional (1-D) transient flow in compliant vessels at the largest scale [4], to unsteady three-dimensional (3-D) flows in curved and flexing vessels at the millimeter range [5], to multimicron-scale thrombus formation at a fissure in the lumen of such a vessel with an atherosclerotic plaque [6]. Further, there are changes over short times (seconds and minutes) in the behavior of platelet structure, receptors, and bonds in a developing thrombus-wall interaction that may or may not lead to embolization. Much is known about the panoply of scales and phenomena involved, but a multiscale modeling needs to be developed to link them.

In the multiscale modeling of blood flow and thrombus formation in arteries, we have used a step-by-step approach in raising computational complexity. By using experimental observations of Begent and Born [7] and Petrishchev and Mikhailova [8] on the effects of blood flow rate on thrombus formation as a target of modeling, we first demonstrated that our model of platelet activation with a delay time provided a variation in short-time thrombus growth rates, which agreed qualitatively with the results of in vivo experiments [6]. We

Digital Object Identifier 10.1109/MEMB.2009.931788 expected some shifts to occur when we treated the blood flow (other than the individual platelets) not as a continuum fluid but with a separate representation of red cells in the plasma [9]. In view of the much higher number density of red cells in blood compared with that in platelets, this would mean keeping track of many more individual cells; additionally, the red cells would need to be modeled with compliance and thus with many nodes to represent the membrane of each individual red cell. While developing a compliant red cell model, we also investigated using a simpler, rigid, and spherical model of the red cell (approximating some pathological cases, such as malaria) at two different concentrations (hematocrits). We expected at least that these would augment the effective diffusivity of platelets as thrombi grew large enough, and this we found. Additionally, with the rigid-sphere model of red cells, we found a jamming effect on red cells upstream of a thrombus and a persistence of higher thrombus growth rates to higher blood flow rates.

In this study, we first briefly review the model that describes platelet behavior resulting in aggregation and thrombus formation. This is followed by an overview of the basics of the dissipative particle dynamics (DPD), the simulation method adopted here. We then describe the details of the numerical simulations and conclude by presenting the results of these simulations.

\section{Platelet Model}

In this study, we use the platelet model previously developed in [6]; see Figure 1 for a schematic explanation. We assume that platelets can be in three different biological states: passive, triggered, and activated. The passive state is a normal state of platelets in blood. Passive platelets are following the flow without any specific interaction with vessel walls or other platelets. When a passive platelet is exposed to a high concentration of activation chemicals (such as adenosine diphosphate), it becomes triggered, and after some time, activated. Triggered platelets are still nonadhesive, whereas activated platelets can adhere to other activated platelets or injured vessel walls when they come sufficiently close to them. The key component of the model is an activation delay time, the time it takes for a platelet to go from passive to activated state. The activation delay time was first proposed in [10]. Based on experimental data, the activation delay time was estimated to be about $0.1-0.2 \mathrm{~s} \mathrm{[10].} \mathrm{In}$ simulations, the diffusion of the activation chemicals released 


\section{The model can also be modified to account for \\ the RBC shape more exactly, also for the \\ compliance of the RBC membrane.}

by platelets is not modeled explicitly, but instead, it is assumed that there is a region surrounding each platelet in which the concentration of chemicals is sufficiently high to trigger the activation of other platelets. This region is represented as a sphere, with the activation center located at the center of the platelet. To account for the uncertainty of the boundaries of the region, the activation delay time for the interacting passive platelet is chosen uniformly at random from a given time interval. The aggregation process involves activated platelets, which are spiny spheres; therefore, spherical shape approximation is used in simulations. The pseudopodia and blood proteins (such as fibrinogen), which adhere to the activated platelets and form links between platelets, are not modeled explicitly. Instead, for each pair of adhered and activated platelets, we assume that there is some range of separation distance where the proteins bridging the platelets are loose. The adhesive force in this range is set to zero. At a larger separation distance, the force gradually increases up to a maximum and consequently decreases to zero, where we assume that all adhesive links between platelets are broken and platelets are completely separated from each other. For a more detailed description of the adhesive forces and other aspects of the model, see [6]. In a more refined multiscale model, we would represent the corresponding cellular/plasmaprotein/adhesion molecule dynamics in greater detail. The model can also be modified to account for the red blood cell (RBC) shape more exactly and also for the compliance of the $\mathrm{RBC}$ membrane.

\section{Simulation Method}

The blood cells and the surrounding fluid are modeled in this study using the DPD method. DPD simulations consist of particles that represent clusters of atoms or molecules [11], [12]. The particles interact with each other through simple, pairwise forces. For simple fluids, there are three types of forces acting on each dissipative particle: a purely repulsive conservative force, a dissipative force that reduces velocity differences between the particles, and a random force directed along the line connecting the centers of the particles. Specifically, the forces exerted on a particle $i$, located at $\mathbf{r}_{i}$ and moving with velocity $\mathbf{v}_{i}$, by particle $j$ are given by the following:

$$
\begin{aligned}
& \mathbf{F}_{i j}^{C}=F^{C}\left(r_{i j}\right) \hat{r}_{i j}, \\
& \mathbf{F}_{i j}^{D}=-\gamma w^{D}\left(r_{i j}\right)\left(\hat{r}_{i j} \cdot \mathbf{v}_{i j}\right) \hat{r}_{i j}, \\
& \mathbf{F}_{i j}^{R}=\sigma w^{R}\left(r_{i j}\right) \xi_{i j} \hat{r}_{i j},
\end{aligned}
$$

where $\mathbf{r}_{i j}=\mathbf{r}_{i}-\mathbf{r}_{j}, r_{i j}=\left|\mathbf{r}_{i j}\right|, \hat{r}_{i j}=\mathbf{r}_{i j} / r_{i j}$, and $\mathbf{v}_{i j}=\mathbf{v}_{i}-\mathbf{v}_{j}$. The variables $\gamma$ and $\sigma$ determine the strength of the dissipative and random forces, respectively. Also, $\xi_{i j}$ are symmetric Gaussian random variables with zero mean and unit variance and are independent for different pairs of particles and at different times; $\xi_{i j}=\xi_{j i}$ is enforced to satisfy the momentum conservation. Finally, $w^{D}$ and $w^{R}$ are weight functions. All forces are acting within a sphere of interaction radius $r_{c}$, which is the length scale of the system. The conservative force is given by a soft potential:

$$
\mathbf{F}_{i j}^{C}=a\left(1-r_{i j} / r_{c}\right) \hat{r}_{i j}
$$

The weight functions and the amplitudes of the dissipative and random forces are linked through the fluctuation-dissipation theorem [13]. Specifically, we have that

$$
w^{D}\left(r_{i j}\right)=\left[w^{R}\left(r_{i j}\right)\right]^{2}
$$

and

$$
\sigma^{2}=2 \gamma k_{\mathrm{B}} T
$$

where $T$ is the system temperature and $k_{\mathrm{B}}$ is the Boltzmann constant. The weight function takes the following form:

$$
w^{R}\left(r_{i j}\right)=1-r_{i j} / r_{c} .
$$

The time evolution of the position and velocity of DPD particle $i$ is described by Newton's equations of motion:

$$
\begin{aligned}
d \mathbf{r}_{i} & =\mathbf{v}_{i} d t, \\
d \mathbf{v}_{i} & =\mathbf{F}_{i}^{C} d t+\mathbf{F}_{i}^{D} d t+\mathbf{F}_{i}^{R} \sqrt{d t},
\end{aligned}
$$

where $\mathbf{F}_{i}^{C}=\sum_{i \neq j} \mathbf{F}_{i j}^{C}$ is the total conservative force acting on particle $i ; \mathbf{F}_{i}^{D}$ and $\mathbf{F}_{i}^{R}$ are defined similarly. A commonly used set of DPD parameters [14] is employed in this study; specifically, the number density of the DPD fluid is $\rho=3$, the conservative force coefficient is $a=25$, and the dissipative and random force coefficients are $\gamma=4.5$ and $\sigma=3$, respectively. The modified velocity Verlet scheme $(\lambda=0.5)$ [14] with time step $d t=0.02$ is used for time integration.

In addition to simulating simple fluids, the DPD method can be extended to complex systems, including colloidal particles and solid surfaces. A typical approach for modeling

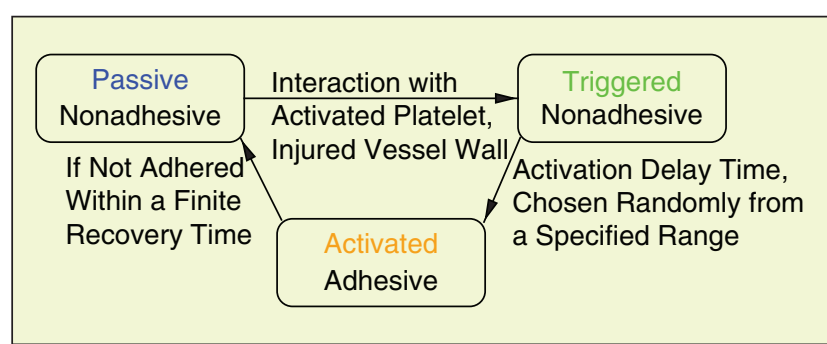

Fig. 1. Schematic explanation of the platelet model. 


\section{The key component of the model is an activation delay time, the time it takes for a \\ platelet to go from passive to activated state.}

solid objects is to group and freeze collections of DPD particles. The particles can be grouped in a specific way to reduce the roughness of the surface [15], [16], be a part of the initial lattice [17], or simply be a subset of the fluid particles [18][20]. In simulations, the motion of these particles is constrained so that their relative positions remain the same. The DPD simulation technique produced realistic rheological behavior for particulate suspensions in previous studies. In [21], both the low- and high-shear viscosities of sphere suspensions up to $30 \%$ were found to be in good agreement with

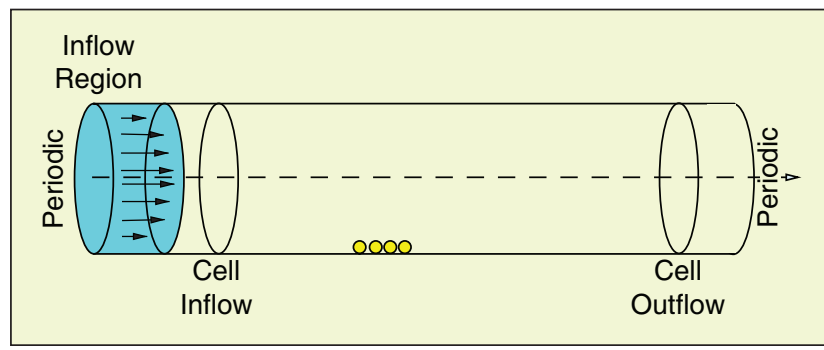

Fig. 2. The simulation domain is a cylinder. The cells are introduced at the inflow plane and removed at the outflow plane. The platelet aggregation is initiated by placing small number of activated platelets attached to the wall.

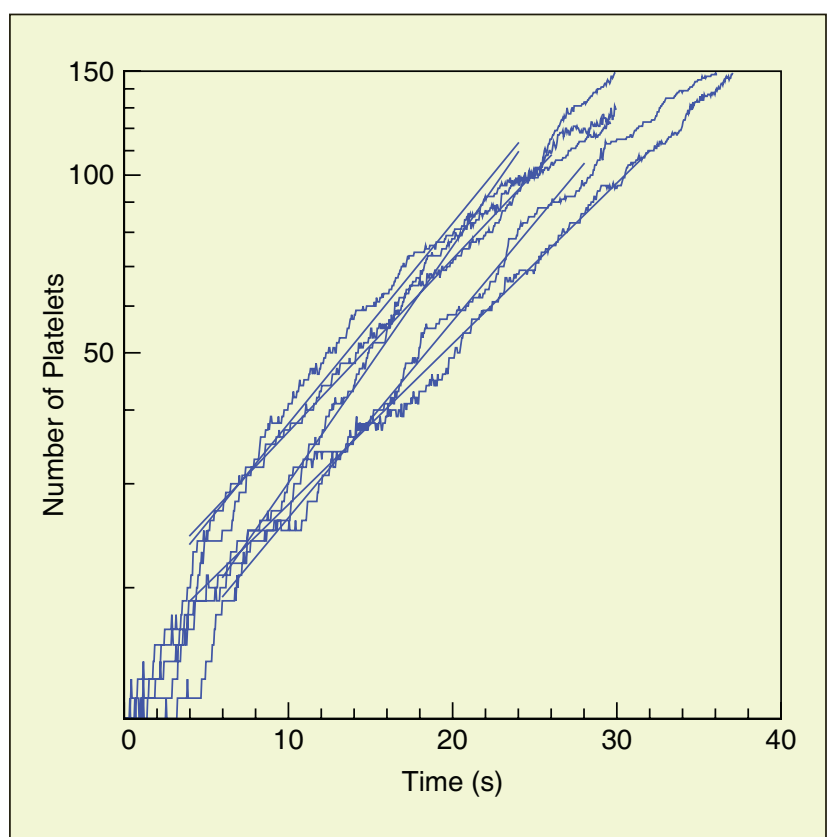

Fig. 3. Accumulation of platelets in a thrombus with a flow rate of $333 \mu \mathrm{m} / \mathrm{s}$ (no RBCs). Solid lines are used to correlate exponential growth phase; slopes are plotted on Figure 4. experimental data. Similar results were obtained in [17]. The flow behavior past a single sphere was investigated in [15]; the DPD simulation results agreed with the results of the previous experiment and lattice-Boltzmann simulation.

The DPD simulations are performed in nondimensional units; therefore, it is necessary to define the DPD scales. We set the unit of length $\left(r_{c}\right)$ in DPD to be $0.72 \mu \mathrm{m}$. Each fluid particle then corresponds to approximately $1.24 \times 10^{-16} \mathrm{~kg}$ of liquid water. The time scale is defined as follows. For a platelet flowing in a vessel of circular cross section, a product of the platelet activation delay time and the velocity gradient at the vessel wall is a nondimensional number. For example, for a platelet with activation delay time of $0.05 \mathrm{~s}$ in a vessel of $30 \mu \mathrm{m}$ in diameter with the flow velocity of $300 \mu \mathrm{m} / \mathrm{s}$ in the center of the vessel, this number is equal to 2 . We define the DPD unit of time in our simulations so that this nondimensional number for given flow velocity in the vessel of equal size is preserved. Specifically, one time unit in our simulations is equal to $0.00216 \mathrm{~s}$. The rest of the parameters and the results presented further in the text are based on this choice of DPD length and time scales.

The vessel (e.g., an arteriole) is modeled as a cylinder, $30 \mu \mathrm{m}$ in diameter (Figure 2). The axis of the cylinder is

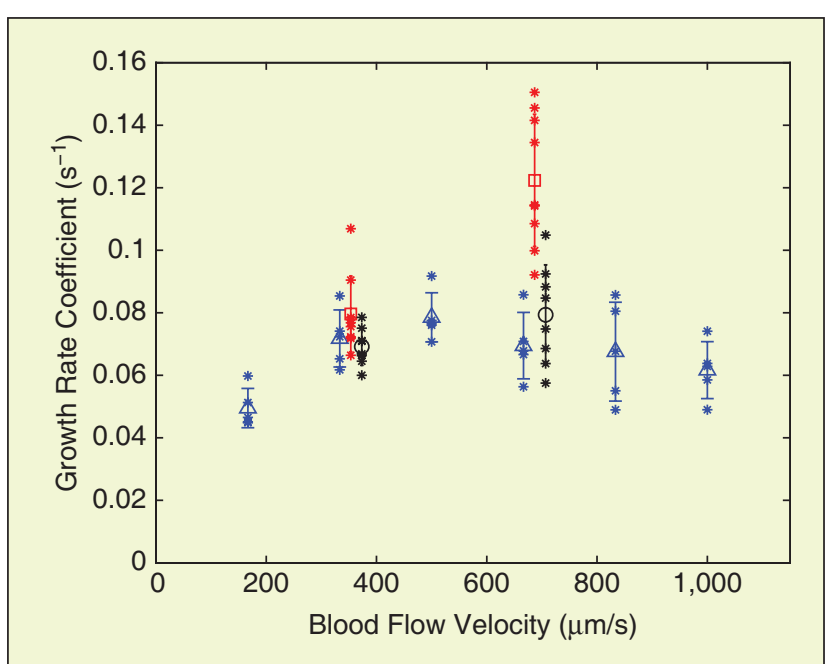

Fig. 4. Exponential thrombus growth rate coefficients as a function of flow rate in a $30-\mu \mathrm{m}$ diameter vessel. The individual values from simulations with different random number sequences are indicated by asterisks. The mean values for simulations without RBC, with $30 \%$ and with $15 \%$ volume fraction of RBCs are shown by triangles, squares, and circles, respectively. For clarity, the results with RBCs are slightly shifted to the right. The trend of the growth rate coefficients as a function of flow rate matches qualitatively the trend from the Begent and Born experiment (7). 
parallel to $z$ coordinate axis and is passing through the origin of the coordinate system. The cylinder extends from $z=0 \mu \mathrm{m}$ to $z=150 \mu \mathrm{m}$. The solid walls are modeled by freezing layers of DPD particles in combination with bounce-back reflection [16] and adaptive boundary conditions [22] at the fluid-solid interface. The system is periodic in $z$ direction; the DPD particles leaving the computational domain at $z=150 \mu \mathrm{m}$ are introduced back into the system at $z=0 \mu \mathrm{m}$. The Poiseuille flow velocity profile can be obtained using external body force; however, to eliminate any potential periodic effects, an alternative approach is used in simulations. The parabolic inflow velocity profile is generated by placing the inflow region inside the domain. The $z$ component of the velocity of every DPD particle inside the inflow region is set to the value equal to the fluid velocity given by the Poiseuille flow solution. The inflow region covers part of the flow domain between $z=0 \mu \mathrm{m}$ and $z=2 \mu \mathrm{m}$.

The platelets and RBCs are modeled as rigid spheres of radii 1.5 and $2.25 \mu \mathrm{m}$, respectively. It may be expected that depletion effects [23], in particular inside the platelet aggregate, may result in an effective attraction. This additional force will add to the attractive forces that are used in the platelet model. This may somewhat affect the results; however, we expect that the effect will be small. In previous simulations [6], we investigated the sensitivity of aggregation process to particular values of attractive force and found that the effect is small for a wide range of parameters. The platelet aggregation is initiated by placing a small number of activated seed platelets attached to the vessel wall. In simulations described here, 12 seed platelets were used. Except for the seed platelets, the flow domain is initially free of cells. We assume that platelets and RBCs are distributed uniformly in the flow upstream of the growing aggregate. During the simulation, platelets and $\mathrm{RBCs}$ are introduced into the flow in $z=7.5 \mu \mathrm{m}$ plane. The probability of adding a new cell at some specific location is proportional to the velocity at this location, which has a parabolic profile. The probabilities are chosen so that the concentration of platelets in simulations is approximately $600,000 / \mathrm{mm}^{3}$. For the volume occupied by the RBCs, two possibilities were investigated as described further in the text. The platelet count was kept on a higher side because the growth rate of thrombus is proportional to the concentration in the approaching stream, and so less time is required to reach a given size than with a lower concentration. In addition, the activation delay time is also reduced in simulations. Specifically, an activation delay time for each platelet is chosen uniformly at random between 0.025 and $0.075 \mathrm{~s}$. When the location for the cell is selected, all fluid particles, which fall inside the volume occupied by the cell, are grouped and their further motion is constrained, so that they move

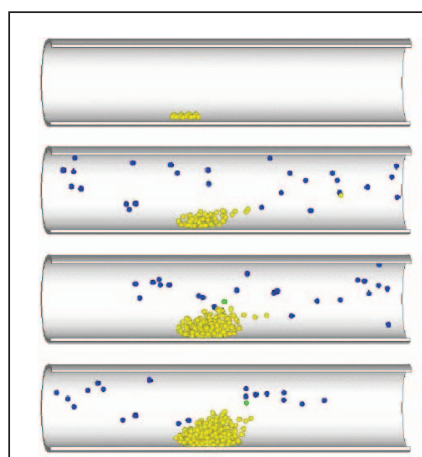

(a)

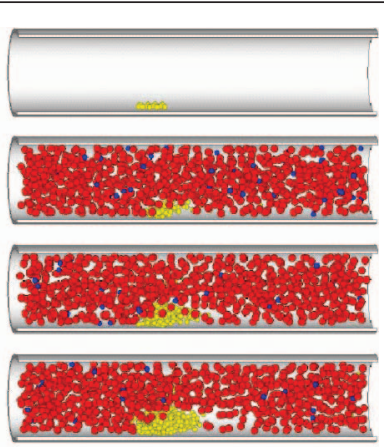

(b)
Fig. 5. Accumulation of platelets in a thrombus in simulations (a) without RBCs and (b) with RBCs. Snapshots are taken at 0 (upper row), 14, 28, and 42 (lower row) s. Passive platelets are shown in blue, triggered in green, and activated in yellow. The RBCs are shown in red. Blood flow velocity is $333 \mu \mathrm{m} / \mathrm{s}$.

as a rigid object. The freshly added platelets and RBCs are allowed to follow the flow and interact with the walls and other cells following the rules described in [6]. Platelets and RBCs reaching the $z=150 \mu \mathrm{m}$ plane are eliminated from the simulations. This is done by removing the constraints on the motion of the DPD particles which form them.

\section{Results}

We first present the results of platelet aggregation in simulations without RBCs. Platelet aggregation at six different blood flow velocities from 166 to $1,000 \mu \mathrm{m} / \mathrm{s}$ was investigated. At each flow velocity, several cases were simulated with different random number generator sequence. The number of platelets in the aggregate as a function of time for five different cases at blood flow velocity $333 \mu \mathrm{m} / \mathrm{s}$ is shown in Figure 3 . There is some variation in platelet aggregation, primarily because of the different distribution of the platelets at the inflow and also because of the random choice of the activation delay time for each individual platelet. In all cases, the aggregation process is characterized by two stages. At the first

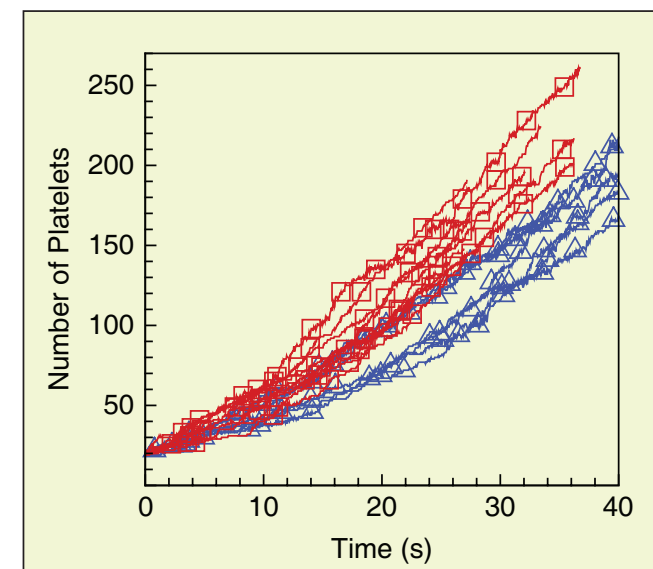

(a)

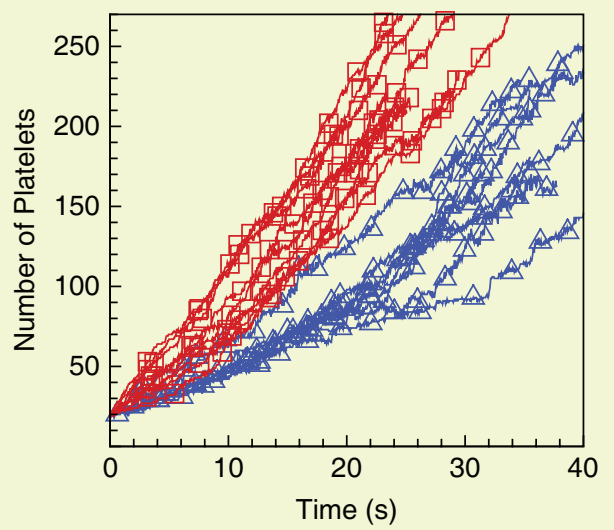

(b)

Fig. 6. Accumulation of platelets in a thrombus. The results without and with RBCs are shown with triangles and squares, respectively. Blood flow velocity is (a) $333 \mu \mathrm{m} / \mathrm{s}$ and (b) $666 \mu \mathrm{m} / \mathrm{s}$. For better comparison, all curves are shifted, so that the time is measured from the moment when the aggregate reaches the size of 20 platelets. 


\section{DPD simulations consist of particles that}

represent clusters of atoms or molecules.

stage, the aggregate consists of small number of platelets (less than approximately 20). The rate of increase of number of platelets in the aggregate at this stage varies quite significantly between different cases. At the second stage, when the aggregate is sufficiently large (more than 20 platelets), an exponential growth of the aggregate is observed in all simulations. We characterize the growth rate by computing the coefficient in the exponent for the aggregates between 20 and 120 platelets in size. For each blood flow velocity, the average growth rate coefficient and the standard deviation were computed. The results are shown in Figure 4. In agreement with experimental data [7], [8] and previous simulations [6], there is an initial increase of the exponential growth rate coefficient with blood flow velocity, followed by a subsequent decrease.

The platelet aggregation is affected by the presence of rigid spheres, which represent RBCs in simulations. In Figure 5, we show snapshots from typical simulations at blood flow velocity of $333 \mu \mathrm{m} / \mathrm{s}$. The RBCs occupy $30 \%$ of the fluid volume at the inflow in these simulations. The shape and the growth rate of small aggregates is not affected significantly by the presence of RBCs. This is also illustrated in Figure 6, where we plot the number of platelets in the aggregate as a function of time. We observe an increase in platelet aggregation when the aggregate grows larger. This is likely due to the enhanced diffusion of platelets in the presence of RBCs, which facilitates the aggregation as the size of the thrombi in the streamwise direction increases. The growth coefficient measured for aggregates of size from 20 to 120 platelets is shown in Figure 4. At $666 \mu \mathrm{m} / \mathrm{s}$, the shape of the aggregate and the growth rate are affected significantly by the presence of RBCs even for small aggregates, as shown in Figures 6 and 7. We believe the

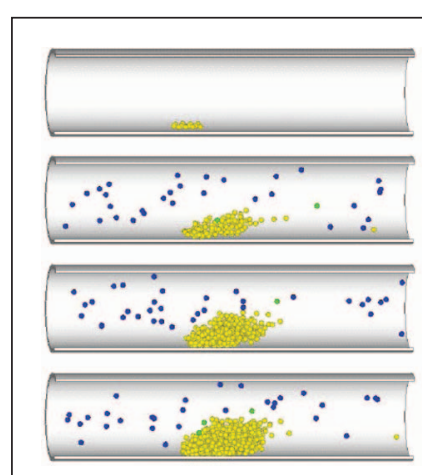

(a)

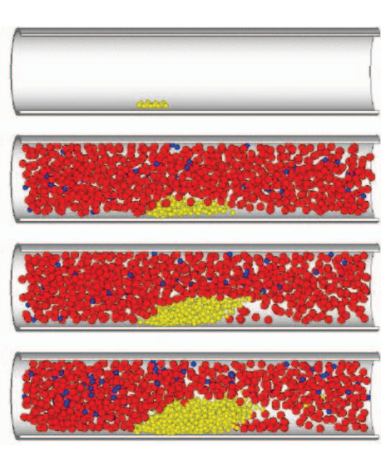

(b)
Fig. 7. Accumulation of platelets in a thrombus in simulations (a) without RBCs and (b) with RBCs. Snapshots are taken at 0 (upper row), 14, 28, and 42 (lower row) s. Passive platelets are shown in blue, triggered in green, and activated in yellow. The RBCs are shown in red. Blood flow velocity is $666 \mu \mathrm{m} / \mathrm{s}$. reason for this is a jamming of RBCs upstream of the aggregate, which can be noticed in Figure 7 and is illustrated in Figure 8 , where we plot the volume fraction of platelets and RBCs for the cases shown in Figures 5 and 7. The jamming results in decrease of RBCs and platelets velocity in the vicinity of aggregate and effectively increases the time of interaction of flowing platelets with the aggregate. This situation may be relevant to some extent in disease conditions, such as malaria, when the RBCs stiffness is greatly increased. To prevent the jamming, we decrease the RBCs concentration, so that they occupy only $15 \%$ of the volume at the inflow. In the absence of jamming, the effect of the presence of RBCs on small aggregate growth rate is not significant as shown in Figure 4.

\section{Conclusions}

In this study, we considered the effect of presence of RBCs on platelet aggregation. We represented the RBCs as rigid spheres in simulations. We found that an enhanced effective diffusivity of platelets in the presence of RBCs increases the growth rate for large aggregates. At 30\% volume fraction of RBCs and blood velocity of $666 \mu \mathrm{m} / \mathrm{s}$, we observed jamming of RBCs upstream of the growing aggregate. As a result, the platelet aggregation was greatly increased. At $15 \%$ RBCs volume

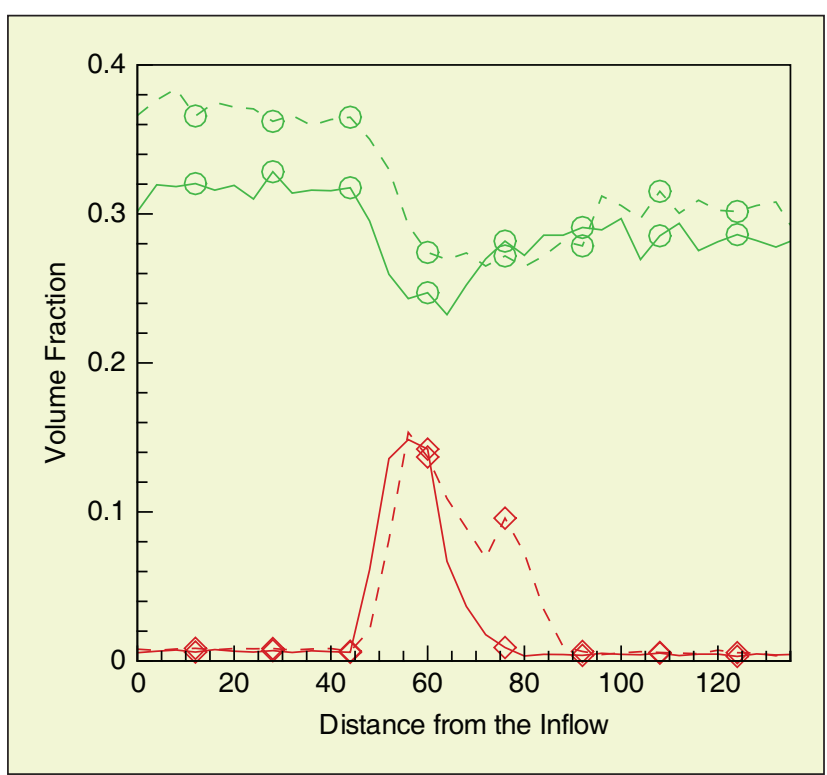

Fig. 8. Volume fraction of platelets (shown with diamonds) and RBCs (shown with circles) as a function of distance from the inflow plane. Results for blood flow velocity $333 \mu \mathrm{m} / \mathrm{s}$ and $666 \mu \mathrm{m} / \mathrm{s}$ are shown with solid and dashed lines, respectively. The distribution of the RBCs varies in time and depends on the size of the platelet aggregate. The profiles are shown for the time moments with aggregates of equal height. 
fraction, the jamming effect was not observed, and the growth rates of small aggregates were close to those computed in the absence of RBCs. The present results of our simulations suggest that $\mathrm{RBC}$ deformability should be taken into account in future investigations, and we are working currently toward this goal.

\section{Acknowledgments}

This work was supported by the NSF/IMAG, and computations were performed at the SDSC Blue Gene (an NSF supercomputing center).

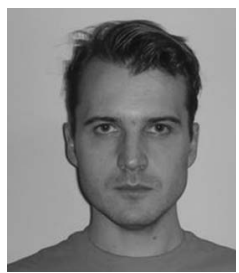

Igor V. Pivkin received his Ph.D. degree in applied mathematics from Brown University. He is currently a postdoctoral research associate in the Department of Materials Science and Engineering at Massachusetts Institute of Technology (MIT). His research interests include biophysics, cellular and molecular biomechanics, stochastic multiscale modeling, and coarse-grained molecular simulations.

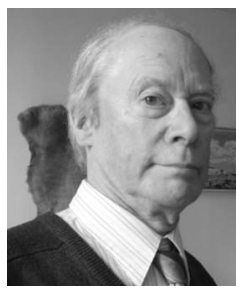

Peter D. Richardson was educated at Imperial College of the University of London and has Ph.D., D.Sc. (Eng), and D.Sc. (physiology) degrees. He is a fellow of the Royal Society, of the American Society of Mechanical Engineers, a founding fellow of the American Society for Medical and Biological Engineering, and an inaugural fellow of the Biomedical Engineering Society. He is a laureate in medicine of the Ernst Jung Foundation. He received HumboldtPreis of the A. von Humboldt Stiftung. Since 1984, he has been professor of engineering and physiology at Brown University.

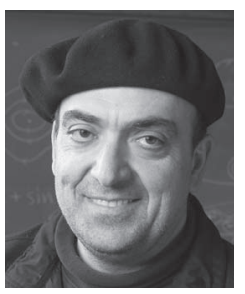

George Em Karniadakis received his B.Sc. degree from National Technical University (NTU), Athens, Greece, in 1982, his M.S. and Ph.D. degrees from MIT in 1984 and 1987, respectively. He was an assistant professor at Princeton University from 1988 to 1993, a visiting professor at California Institute of Technology (Caltech) in the Spring quarter of 1993, an associate professor at Brown University from 1994 to 1996, and became a full professor at Brown University in 1996. He has been a visiting professor and senior lecturer of mechanical engineering at the MIT since 2000 . He developed the first algorithms for microfluidics in the late 1980s and published the first book on this field. He pioneered parallel computing in 3-D turbulence simulations, organized several international conferences, and developed and maintained the code NEKTAR (freeware) used in Universities, national labs, and industry. His work has appeared on the covers of many scientific journals. He has authored three books and more than 200 research papers. He has received research support from the Office of Naval Research, the National Science Foundation, the U.S. Air Force Office of Scientific Research, and the U.S.-Israel Binational Science Foundation.
He is a fellow of the American Society of Mechanical Engineers, a fellow of the American Physical Society, and an AIAA associate fellow. In 2007, he was awarded the Computational Fluid Dynamics Award presented by the U.S. Association of Computational Mechanics.

Address for Correspondence: Igor V. Pivkin, Department of Materials Science and Engineering, MIT, 77 Massachusetts Avenue, Cambridge, MA 02139 USA. E-mail: piv@mit.edu.

\section{References}

[1] A. Donne, "De L'origine des globules du sang, de leur mode de formation et de leur fin," Comp. Rend. Acad. Sci., vol. 14, p. 366, 1842.

[2] G. Bizzozero, "Uber einen neuen Formbestandteil des Bluites und dessen Rolle bei der Thrombose und der Blutgerinnung," Virchows Arch. Pathol. Anat. Physiol., vol. 90, p. 261, 1882

[3] S. M. Dopheide, C. L. Yap, and S. P. Jackson, "Dynamic aspects of platelet adhesion under flow," Clin. Exp. Pharmacol. Physiol., vol. 28, no. 5-6, pp. 355363,2001

[4] S. J. Sherwin, L. Formaggia, J. Peiro, and V. Franke, "Computational modelling of 1D blood flow with variable mechanical properties and its application to the simulation of wave propagation in the human arterial system," Int. J. Numer. Methods Fluids, vol. 43, no. 6-7, pp. 673-700, 2003.

[5] I. V. Pivkin, P. D. Richardson, D. H. Laidlaw, and G. E. Karniadakis, "Combined effects of pulsatile flow and dynamic curvataure on wall shear stress in cornonary artery bifurcation model," J. Biomech., vol. 38, no. 6, pp. 1283-1290, 2005. [6] I. V. Pivkin, P. D. Richardson, and G. Karniadakis, "Blood flow velocity effects and role of activation delay time on growth and form of platelet thrombi," Proc. Natl. Acad. Sci. USA, vol. 103, no. 46, pp. 17164-17169, 2006.

[7] N. Begent and G. V. Born, "Growth rate in vivo of platelet thrombi, produced by iontophoresis of ADP, as a function of mean blood flow velocity," Nature, vol. 227 , no. 5261, pp. 926-30, 1970 .

[8] N. N. Petrishchev and I. A. Mikhailova, "Influence of some hydrodynamic factors of thrombus formation in microvessels," Microvasc. Res., vol. 49, no. 1, pp. 12-6, 1995.

[9] E. C. Eckstein and F. Belgacem, "Model of platelet transport in flowing blood with drift and diffusion terms," Biophys. J., vol. 60, no. 1, pp. 53-69, 1991.

[10] P. D. Richardson, "Effect of blood flow velocity on growth rate of platelet thrombi," Nature, vol. 245, no. 5420, pp. 103-104, 1973.

[11] P. J. Hoogerbrugge and J. M. V. A. Koelman, "Simulating microscopic hydrodynamic phenomena with dissipative particle dynamics," Europhys. Lett. vol. 19, no. 3, pp. 155-160, 1992.

[12] E. E. Keaveny, I. V. Pivkin, M. Maxey, and G. E. Karniadakis, "A comparative study between dissipative particle dynamics and molecular dynamics for simple- and complex-geometry flows," J. Chem. Phys., vol. 123, no. 10, p. 104107, 2005.

[13] P. Espanol and P. Warren, "Statistical-mechanics of dissipative particle dynamics," Europhys. Lett., vol. 30, no. 4, pp. 191-196, 1995.

[14] R. D. Groot and P. B. Warren, "Dissipative particle dynamics: Bridging the gap between atomistic and mesoscopic simulation," J. Chem. Phys., vol. 107, no. 11, pp. 4423-4435, 1997

[15] S. Chen, N. Phan-Thien, B. C. Khoo, and X. J. Fan, "Flow around sphere by dissipative particle dynamics," Phys. Fluids, vol. 18, no. 10, p. 103605, 2006.

[16] I. V. Pivkin and G. E. Karniadakis, "A new method to impose no-slip boundary conditions in dissipative particle dynamics," J. Comput. Phys., vol. 207, no. 1, pp. 114-128, 2005.

[17] N. S. Martys, "Study of a dissipative particle dynamics based approach for modeling suspensions," J. Rheol., vol. 49, no. 2, pp. 401-424, 2005.

[18] E. S. Boek, P. V. Coveney, H. N. W. Lekkerkerker, and P. van der Schoot, "Simulating the rheology of dense colloidal suspensions using dissipative particle dynamics," Phys. Rev. E, vol. 55, no. 3, pp. 3124-3133, 1997.

[19] Y. Kong, C. W. Manke, W. G. Madden, and A. G. Schlijper, "Simulation of a confined polymer in solution using the dissipative particle dynamics method," Int. J. Thermophys., vol. 15, no. 6, pp. 1093-1101, 1994

[20] D. A. Fedosov, I. V. Pivkin, and G. E. Karniadakis, "Velocity limit in DPD simulations of wallbounded flows," J. Comput. Phys., vol. 227, no. 4, pp. 2540 2559,2008

[21] E. S. Boek, P. V. Coveney, and H. N. W. Lekkerkerker, "Computer simulation of rheological phenomena in dense colloidal suspensions with dissipative particle dynamics," J. Phys.-Condensed Matter, vol. 8, no. 47, pp. 9509-9512, 1996.

[22] I. V. Pivkin and G. E. Karniadakis, "Controlling density fluctuations in wallbounded dissipative particle dynamics systems," Phys. Rev. Lett., vol. 96, no. 20, p. 206001, 2006

[23] E. S. Boek and P. van der Schoot, "Resolution effects in dissipative particle dynamics simulations," Int. J. Modern Phys. C, vol. 9, no. 8, pp. 1307-1318, 1998. 